\title{
Ethyl acetate extract from Inula helenium L. inhibits the proliferation of pancreatic cancer cells by regulating the STAT3/AKT pathway
}

\author{
BO ZHANG ${ }^{1}$, JIANMEI ZENG ${ }^{1,2}$, YOUYOU YAN ${ }^{1}$, BO YANG $^{2}$, MINCONG HUANG ${ }^{2}$, \\ LINLING WANG $^{1,2}$, QI ZHANG ${ }^{1}$ and NENGMING LIN ${ }^{1,2}$
}

${ }^{1}$ Translational Medicine Research Center, Hangzhou First People's Hospital, Nanjing Medical University, Hangzhou, Zhejiang 310006; ${ }^{2}$ College of Pharmaceutical Sciences, Zhejiang Chinese Medical University, Hangzhou, Zhejiang 310053, P.R. China

Received October 6, 2017; Accepted December 21, 2017

DOI: $10.3892 / \mathrm{mmr} .2018 .8534$

\begin{abstract}
Sesquiterpene lactones are bioactive compounds that have been identified as responsible for the anticancer activity of the medicinal herb, Inula helenium L. (IHL). However, the mechanisms of action involved in the anti-pancreatic cancer activity of IHL have yet to be elucidated. The present study used an optimized extraction strategy to obtain sesquiterpene lactones from IHL (the resulting product termed ethyl acetate extract of IHL; EEIHL), and examined the potential mechanisms involved in the anti-pancreatic cancer activity of EEIHL. Ethanol and ethyl acetate were used to extract sesquiterpene lactones from IHL to give the final product EEIHL. Cell Counting Kit-8, colony formation and Annexin V/propidium iodide assays were used to detect the anti-proliferative activity of EEIHL. Cell migration was determined with a wound healing assay. mRNA and protein expression levels were analyzed by reverse transcription-quantitative polymerase chain reaction and western blot analyses, respectively. It was identified that low concentrations of EEIHL caused CFPAC-1 cell cycle arrest in the $G_{0} / G_{1}$ phase, whereas high concentrations of EEIHL induced mitochondria-dependent apoptosis. In addition, EEIHL could inhibit the phosphorylation of the signal transducer and activator of transcription (STAT)3/AKT pathway, potentially resulting in impeded cell mobility. In conclusion, EEIHL could activate mitochondrial-dependent apoptosis and inhibit cell migration through the STAT3/AKT pathway in CFPAC-1 cells.
\end{abstract}

Correspondence to: Professor Nengming Lin, Translational Medicine Research Center, Hangzhou First People's Hospital, Nanjing Medical University, 261 Huansha Road, Hangzhou, Zhejiang 310006, P.R. China

E-mail:1nm1013@163.com

Key words: Inula helenium L., pancreatic cancer, cell cycle, apoptosis, signal transducer and activator of transcription 3/AKT pathway

\section{Introduction}

Pancreatic ductal adenocarcinoma (PDAC) is one of the most aggressive malignancies and may become the second leading cause of cancer-associated mortality by the year 2030 (1). The majority of PDAC patients are diagnosed at advanced and aggressive stage, when surgery is not an option (2). Therefore, systemic chemotherapy is crucial for PDAC therapy at advanced stages (3). Although great efforts have been dedicated into developing efficacious compounds against PDAC in preclinical and clinical studies, the 5-year median survival rate remains at $\sim 5 \%$ and has not changed significantly during the past 40 years (4). Thus, novel therapeutic compounds are urgently required to combat this aggressive malignancy.

Inula helenium L. (IHL) is a traditional Chinese medicinal herb that has been used to treat intestinal tuberculosis associated-enterorrhagia, bronchitis and chronic enterogastritis, and has recently been identified to possess anticancer activity (5-7). Sesquiterpene lactones are a family of compounds that being identified responsible for the anticancer activity of IHL (8-10). However, existing methods have proved to be insufficient to extract sesquiterpene lactones from IHL. Ineffective components in the IHL crude extract would undermine the anti-cancer activity (11-13). In addition, the anti-proliferative activity of IHL against PDAC cells has yet to be reported.

The present study optimized the extraction process of IHL. The dried rhizome and root of IHL was first extracted with $95 \%$ ethanol, and yielded extracts were partitioned by ethyl acetate to give the final product, ethyl acetate extract of IHL (EEIHL). It was identified that EEIHL induced CFPAC- 1 cell cycle arrest in the $\mathrm{G}_{0} / \mathrm{G}_{1}$ phase, and induced mitochondria-dependent apoptosis. In addition, EEIHL demonstrated potent anti-migration activity compared with isoalantolactone, possibly through downregulating the phosphorylation of signal transducer and activator of transcription (STAT)3 and AKT, thus indicating that EEIHL may possess anti-proliferative activity against PDAC cells. 


\section{Materials and methods}

Extraction and characterization of EEIHL. Inula helenium $\mathrm{L}$. was obtained from the Bozhou traditional Chinese medicine market (Anhui, China). The dried rhizome and root of Inula helenium L. (10.0 kg) were crushed into coarse powder. The powder was soaked with $95 \%$ ethanol for $48 \mathrm{~h}$, and extracted with 20 -fold $95 \%$ ethanol at room temperature by percolation. The combined extract was concentrated in a vacuum to give a crude extract. The crude extract was suspended in 1.51 warm water and partitioned with ethyl acetate $(3 \times 1.51)$. Subsequent to evaporation in reduced pressure, $370.16 \mathrm{~g}$ EEIHL was yielded (yield, 3.7\%). To verify that EEIHL contained bioactive compounds, a vanillin-sulfuric acid colorimetry assay was applied to establish sesquiterpene lactones in EEIHL (14).

High performance liquid chromatography (HPLC) chromatogram of EEIHL. EEIHL was run through an Acquity UPLC system with a BEH C18 column $\left(25^{\circ} \mathrm{C}, 2.1 \times 100 \mathrm{~mm}\right.$, $1.7 \mu \mathrm{m}$; Waters Corporation, Milford, MA, USA). $\mathrm{CH}_{3} \mathrm{CN}$ and $\mathrm{H}_{2} \mathrm{O}$ were used as the mobile phase, and a gradient program was applied as follows: 0-2.5 min, $30 \%$ of $\mathrm{CH}_{3} \mathrm{CN} ; 2.5-4 \mathrm{~min}$, $30-45 \%$ of $\mathrm{CH}_{3} \mathrm{CN} ; 4-7 \mathrm{~min}, 45 \%$ of $\mathrm{CH}_{3} \mathrm{CN} ; 7-8 \mathrm{~min}, 45-60 \%$ of $\mathrm{CH}_{3} \mathrm{CN}$; $8-9 \mathrm{~min}, 60 \%$ of $\mathrm{CH}_{3} \mathrm{CN} ; 9-10 \mathrm{~min}, 60-85 \%$ of $\mathrm{CH}_{3} \mathrm{CN} ; 10-12 \mathrm{~min}, 85 \%$ of $\mathrm{CH}_{3} \mathrm{CN} ; 12-13 \mathrm{~min}, 85-30 \%$ of $\mathrm{CH}_{3} \mathrm{CN}$; and maintained at $30 \%$ of $\mathrm{CH}_{3} \mathrm{CN}$ for the next $3 \mathrm{~min}$. The sample solution was $5 \mathrm{mg}$ of EEIHL dissolved in $1.0 \mathrm{ml}$ $\mathrm{MeOH}$, and the injection volume was $1 \mu \mathrm{l}$ with the flow rate of $0.3 \mathrm{ml} / \mathrm{min}$. The detection wavelength was set at $210 \mathrm{~nm}$.

Proton nuclear magnetic resonance $\left({ }^{1} H-N M R\right)$ analysis of EEIHL. The presence of alantolactone, isoalantolactone and alloalantolactone was verified by ${ }^{1} \mathrm{H}-\mathrm{NMR}$. A total of $10 \mathrm{mg}$ of sample was dissolved in $\mathrm{CDCl}_{3}$, and then detected by NMR. Tetramethylsilane was used as internal standard. The ${ }^{1} \mathrm{H}-\mathrm{NMR}$ was performed with a Bruker Ultra Shield Plus 600 $\mathrm{MHz}$ spectrometer (Bruker Corporation, Billerica, MA, USA).

Materials and cell culture. RPMI-1640 medium and fetal bovine serum (FBS) were purchased from Gibco (Thermo Fisher Scientific, Inc., Waltham, MA, USA). The Cycletest Plus DNA Reagent kit was purchased from BD Biosciences (Franklin Lakes, NJ, USA). DAPI was purchased from Beyotime Biotechnology (Shanghai, China). The Annexin V-FITC Apoptosis kit was purchased from BestBio Company (Shanghai, China). A Mitochondrial Membrane Potential Assay kit was purchased from Signalway Antibody LLC (College Park, MD, USA).

The primary antibodies against cyclin-dependent kinase (CDK)2 (cat. no. ab32147), phosphorylated (p)-CDK2 (cat. no. ab76146), CDK6 (cat. no. ab124821), cyclin D1 (cat. no. ab134175), p-Rb (cat. no. ab184796), cyclin E1 (cat. no. ab135380), Bcl-2 (cat. no. ab32124), Bax (cat. no. ab32503), Bim (cat. no. ab32158), Mcl-1 (cat. no. ab32087), poly(ADP-ribose) polymerase (PARP; cat. no. ab191217), caspase-3 (cat. no. ab13847), X-linked inhibitor of apoptosis protein (XIAP; cat. no. ab21278), AKT (cat. no. ab8805), p-AKT (cat. no. ab38449), signal transducer and activator of transcription 3 (STAT3; cat. no. ab68153), p-STAT3 (cat. no. ab76315) and $\beta$-actin (cat. no. ab8226) were purchased from Abcam (Cambridge, MA, USA). Primary antibodies were diluted at 1:1,000.

CFPAC-1 human PDAC cells were purchased from the Cell Bank of the Chinese Academy of Sciences (Shanghai, China). Cells were cultured with RPMI-1640 medium containing $10 \%$ FBS and $1 \%$ penicillin/streptomycin at $37^{\circ} \mathrm{C}$ in a $5 \% \mathrm{CO}_{2}$ humidified atmosphere. EEIHL was dissolved in DMSO at $100 \mathrm{mg} / \mathrm{ml}$.

Cell viability assay. The rate of cell proliferation was measured by a Cell Counting Kit-8 (CCK-8) assay (BestBio Company). Cells were cultured in 96-well plates at a concentration of $7 \times 10^{3} /$ well. Cells were cultured for $24 \mathrm{~h}$ and treated with the indicated concentrations of EEIHL. At $48 \mathrm{~h}$ of treatment, the supernatant was removed, $100 \mu \mathrm{l}$ of CCK- 8 solution was added to each well and the plates were incubated for a further $2 \mathrm{~h}$ at $37^{\circ} \mathrm{C}$. Cell viability was quantified by a Multiskan Spectrum spectrophotometer (Thermo Fisher Scientific, Inc.) by the optical density (OD) at $450 \mathrm{~nm}$. Cell viability was calculated as $\left[\left(\mathrm{OD}_{450}\right.\right.$ of treated cells $/ \mathrm{OD}_{450}$ of control cells $\left.) \times 100 \%\right]$. Three independent experiments were performed.

Colony formation assay. CFPAC-1 cells were seeded into 6 -well plates at a density of $1 \times 10^{3} /$ well and incubated for $24 \mathrm{~h}$. The cells were then treated with the indicated concentrations of EEIHL. Following $24 \mathrm{~h}$ of treatment, the supernatant was removed and cells were cultured for a further two weeks. Then the cells were fixed with $4 \%$ paraformaldehyde for $15 \mathrm{~min}$ and stained with Giemsa solution for $15 \mathrm{~min}$ at room temperature. Visible colonies were imaged with a ChemiDoc XPS system (Bio-Rad Laboratories, Inc., Hercules, CA, USA).

Cell cycle analysis. Exponentially growing cells were seeded in 6-well plates $\left(2 \times 10^{5} /\right.$ well $)$ and cultured overnight in a $5 \% \mathrm{CO}_{2}$ atmosphere at $37^{\circ} \mathrm{C}$. Following treatment with EEIHL, CFPAC-1 cells were harvested and washed twice with cold PBS and then fixed in $70 \%$ cold ethanol at $4^{\circ} \mathrm{C}$ overnight. Cells were stained with the Cycletest Plus DNA Reagent kit (BD Biosciences), according to the manufacturer's protocol. Cell cycle distribution was analyzed using a flow cytometer (BD Biosciences). Data were collected by BD FACSDiva software version 8.0.1 (BD Biosciences) and analyzed by ModFit LT for Windows version 4.1.7 (Verity Software House, Topsham, ME, USA). Three independent experiments were performed.

Apoptosis assay. Exponentially growing cells were seeded in 6-well plates $\left(2 \times 10^{5} /\right.$ well $)$ and cultured overnight in a $5 \%$ $\mathrm{CO}_{2}$ atmosphere at $37^{\circ} \mathrm{C}$. Following treatment with EEIHL for $48 \mathrm{~h}$, the cells were harvested and washed with PBS. Then the cells were stained with the Annexin V-FITC Apoptosis kit, according to the manufacturer's protocol, and analyzed with a flow cytometer (BD Biosciences). Three independent experiments were performed.

DAPI staining. CFPAC-1 cells ( $8 \times 10^{4}$ cells/well) were cultured in 24-well plates. Following exposure to EEIHL, cells were fixed with $4 \%$ paraformaldehyde for $20 \mathrm{~min}$, and stained with DAPI for $15 \mathrm{~min}$ at room temperature. Following washing with 
PBS, cells were observed under a fluorescence microscope (Ti-E; Nikon Corporation, Tokyo, Japan).

Detection of mitochondrial membrane potential. Mitochondrial membrane potential was visualized by the 5,5',6,6'-tetrachloro-1,1',3,3' tetraethyl-imidacarbocyanine iodide (JC-1) stain from the Mitochondrial Membrane Potential Assay kit. Cells were seeded into 6-well plates at a density of $2 \times 10^{5} /$ well, and cultured for $24 \mathrm{~h}$. Following treatment, the cells were collected, washed with PBS, and incubated with JC-1 for $15 \mathrm{~min}$ at $37^{\circ} \mathrm{C}$. Subsequent to washing, cells were immediately analyzed using a flow cytometer (BD Biosciences). Three independent experiments were performed.

Caspase -3 activity assay. CFPAC- 1 cells $\left(2 \times 10^{5}\right.$ cells/well, 6-well plate) were incubated with EEIHL for $48 \mathrm{~h}$. Cells were washed with PBS and lysed in cell lysis buffer included in the Caspase-3 assay kit. Caspase-3 activity in cell lysates were determined colorimetrically using a BioVision colorimetric caspase assay kit (BioVision, Inc., Milpitas, CA, USA). Chromophore conjugated peptides, including DEVD-p-nitroanilide (pNA) and VEID-pNA, were substrates for caspase-3, releasing pNA on caspase activity, which was quantified according to the manufacturer's protocol.

Western blot analysis. Following treatment with different concentrations of EEIHL, total proteins $(40 \mu \mathrm{g})$ were extracted using radioimmunoprecipitation assay lysing buffer (RIPA; cat. no. P0013B; Beyotime Institute of Biotechnology, Shanghai, China) and subjected to $12 \%$ SDS-PAGE prior to being transferred onto PVDF membranes (Bio-Rad Laboratories, Inc.). The membranes were blocked with 5\% non-fat milk at room temperature for $1 \mathrm{~h}$, and then incubated with specific primary antibodies overnight at $4^{\circ} \mathrm{C}$. Subsequent to washing in TBST, membranes were incubated with the secondary antibodies (dilution 1:5,000; HRP Goat Anti-Mouse cat. no. A21010 and HRP Goat Anti-Rabbit cat. no. A21020; Abbkine, Wuhan, Hubei, China) at room temperature for a further $1 \mathrm{~h}$. The protein bands were visualized using an ECL system WBKLS0050 (EMD Millipore, Billerica, MA, USA) and analyzed using Bio-Rad Laboratories Quantity One software version 4.6.2 (Bio-Rad Laboratories, Inc., Hercules, CA, USA).

Wound healing assay. Exponentially growing cells were seeded in 6-well plates ( $2 \times 10^{5} /$ well) and cultured overnight in a $5 \% \mathrm{CO}_{2}$ atmosphere at $37^{\circ} \mathrm{C}$. Following a 24-h incubation, a 1-ml pipette tip was used to gently scratch the monolayer at the center of the well. Detached cells were washed away with PBS, and the remaining cells were cultured with serum-free medium and the indicated doses of treatment. Cells were incubated for $24 \mathrm{~h}$ prior to the capture of images (DS-Ri2; Nikon Corporation).

Reverse transcription-quantitative polymerase chain reaction $(R T-q P C R)$. Total RNA was extracted from cells with TRIzol (Life Technologies; Thermo Fisher Scientific, Inc.), precipitated with isopropyl alcohol, and rinsed with $70 \%$ ethanol. Single-strand cDNA was prepared from the purified RNA using PrimeScript RT Master Mix (cat. no. RR036A; Takara Biotechnology Co., Ltd., Dalian, Liaoning, China), followed by

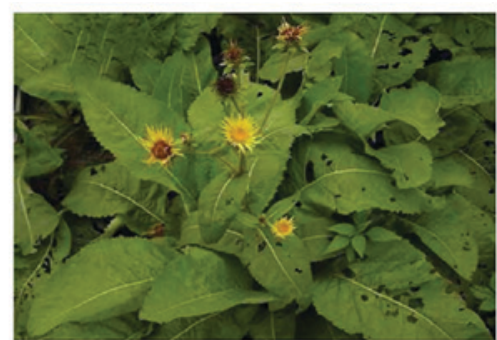

Inula helenium L.
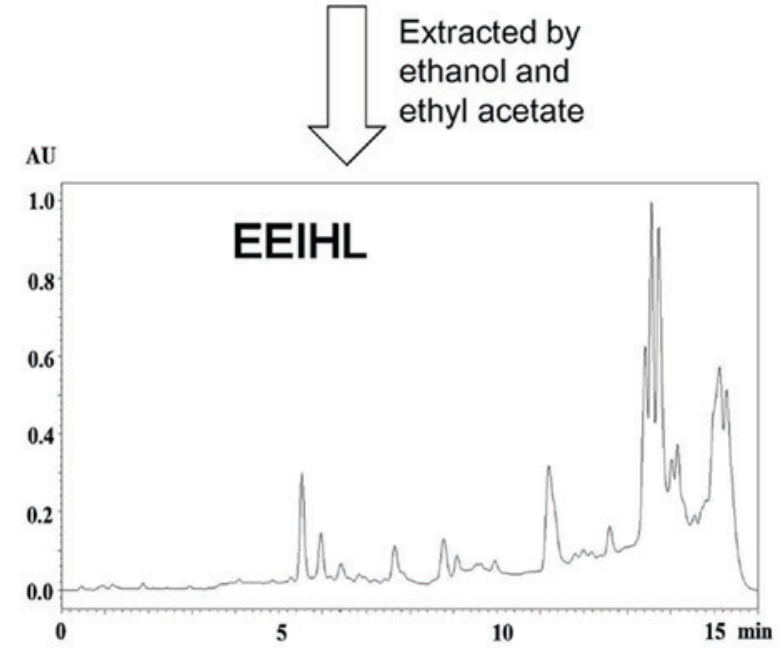

Figure 1. Image of Inula helenium L. Dried rhizomes and roots of Inula helenium L. were extracted with ethanol and partitioned with ethyl acetate. The resulting EEIHL product was analyzed by high performance liquid chromatography. EEIHL, ethyl acetate extract of Inula helenium L.

qPCR with SYBR-Green (Qiagen GmbH, Hilden, Germany) in the 7900HT Fast Real-Time PCR system (Applied Biosystems; Thermo Fisher Scientific, Inc.). The thermocycling parameters were $94^{\circ} \mathrm{C}$ for $30 \mathrm{sec}$ followed by 40 cycles of $58^{\circ} \mathrm{C}$ for $30 \mathrm{sec}$ and $72^{\circ} \mathrm{C}$ for $30 \mathrm{sec}$, with one final cycle of $72^{\circ} \mathrm{C}$ for $90 \mathrm{sec}$. Three independent experiments were performed. The relative expression of target genes against the reference gene was obtained from $2^{-\Delta \Delta C q}$ values (15).

The primers used were: E-Cadherin, forward, 5'-TTCTGC TGCTCTTGCTGTTT-3', reverse, 5'-TGGCTCAAGTCA AAGTCCTG-3'; Snail, forward, 5'-GAAAGGCCTTCAACT GCAAA-3', reverse, 5'-TGACATCTGAGTGGGTCTGG-3'; GAPDH, forward, 5'-GAGTCAACGGATTTGGTCGT-3', reverse, 5'-TTGATTTTGGAGGGATCTCG-3'.

Statistical analysis. The results are expressed as the mean \pm standard deviation of at least three independent experiments. A two-sided Student's t test or one-way analysis of variance followed by a post hoc Fisher's least significant difference test was used to analyze the differences among groups. Graphs were prepared using SigmaPlot software version 12.0 (Systat Software, Inc., San Jose, CA, USA). P<0.05 was considered to indicate a statistically significant difference.

\section{Results}

Characterization of EEIHL. To extract sesquiterpene lactones from crude IHL roots efficiently, ethanol and ethyl acetate were 

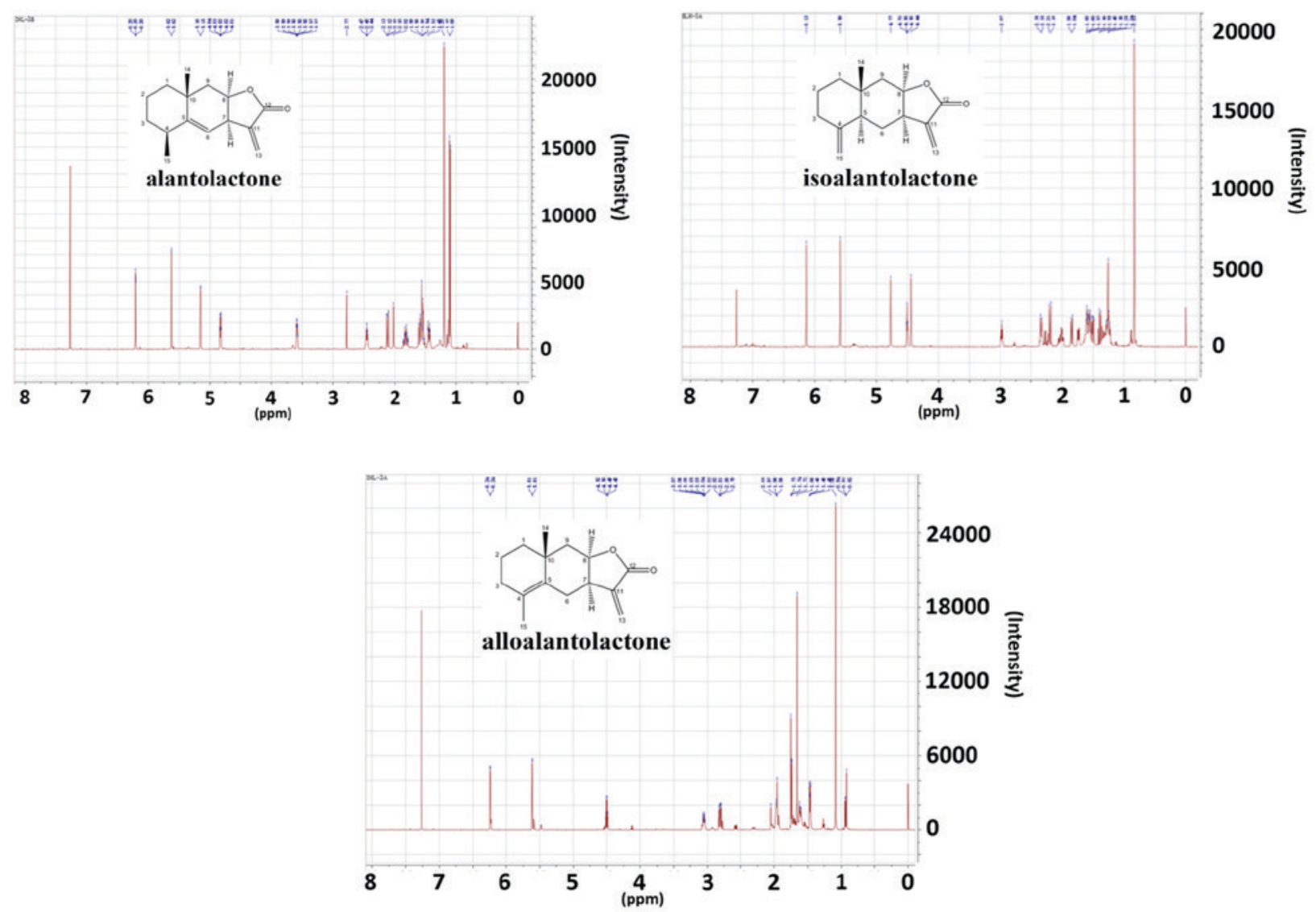

Figure 2. ${ }^{1} \mathrm{H}-\mathrm{NMR}$ of alantolactone, isoalantolactone and alloalantolactone. A total of $10 \mathrm{mg}$ sample was dissolved in $\mathrm{CDCl}_{3}$ and detected with ${ }^{1} \mathrm{H}-\mathrm{NMR}$. The $\delta$ scale is relative to tetramethylsilane, an internal standard, at $\delta=0 .{ }^{1} \mathrm{H}-\mathrm{NMR}$, proton nuclear magnetic resonance spectra.

used to partition IHL and produce the final product, EEIHL. A vanillin-sulfuric acid colorimetry assay was then applied to establish sesquiterpene lactones in EEIHL. The color of the mixture was purple or purple-red, indicating the existence of sesquiterpene lactones in EEIHL, as previously described (16). These three compounds were further confirmed by analysis with HPLC (Fig. 1). In addition, ${ }^{1} \mathrm{H}-\mathrm{NMR}$ spectra were used to identify the presence and structure of alantolactone, isoalantolactone and alloalantolactone (Fig. 2).

EEIHL inhibited the proliferation of CFPAC-1 cells. The anti-proliferative activity of EEIHL against CFPAC-1 PDAC cells was determined by CCK-8 assay and colony formation assay. As shown in Fig. 3A, EEIHL and isoalantolactone dose-dependently inhibited the proliferation of CFPAC-1 cells at $48 \mathrm{~h}$ treatment. The inhibition rate was $>90 \%$ following treatment with $6 \mu \mathrm{g} / \mathrm{ml}$ of EEIHL for $48 \mathrm{~h}$, and the half maximal inhibitory concentration $\left(\mathrm{IC}_{50}\right)$ was 4.3 and $2.8 \mu \mathrm{g} / \mathrm{ml}$ for EEIHL and isoalantolactone, respectively. In addition, a relatively low concentration of EEIHL inhibited the formation of CFPAC-1 colonies (Fig. 3B). When cells were treated with $2 \mu \mathrm{g} / \mathrm{ml}$ of EEIHL for $24 \mathrm{~h}$ and cultured for a further 2 weeks, the number of CFPAC-1 colonies was diminished. At concentrations of 1 or $2 \mu \mathrm{g} / \mathrm{ml}$, EEIHL demonstrated anti-proliferation activity after $24 \mathrm{~h}$ treatment. Therefore, EEIHL potently inhibited the proliferation and colony formation of CFPAC-1 cells.
Low concentrations of EEIHL caused CFPAC-1 cell-cycle arrest at the $G_{0} / G_{1}$ phase. Following treatment with EEIHL for $24 \mathrm{~h}$, CFPAC-1 cells were stained with propidium iodide (PI) followed by cell cycle analysis. As shown in Fig. 4A and B, $2 \mu \mathrm{g} / \mathrm{ml}$ of EEIHL treatment resulted in a $15 \%$ increase in the proportion of cells in the $\mathrm{G}_{0} / \mathrm{G}_{1}$ phase. The protein expression of total CDK2 and p-CDK2 gradually decreased following treatment with EEIHL (Fig. 4C), whereas the protein level of cyclin D1 or cyclin E1 remained unchanged. Notably, pRb was downregulated following EEIHL treatment, indicating that EEIHL may downregulate CDK2 by affecting $\mathrm{pRb}$.

High concentrations of EEIHL caused mitochondrialdependent apoptosis in CFPAC-1 cells. As the EEIHL concentration was increased, CFPAC-1 cells displayed an increasing extent of mitochondrial-dependent apoptosis. As shown in Fig. 5A and B, 4 and $6 \mu \mathrm{g} / \mathrm{ml}$ of EEIHL treatment induced 27 and $48 \%$ apoptosis, respectively, while untreated cells displayed an apoptosis rate of $6 \%$. Meanwhile, $4 \mu \mathrm{g} / \mathrm{ml}$ of EEIHL resulted in shrunken nuclei and intensified DAPI fluorescence (Fig. 5C).

To explore the role of the mitochondria in EEIHL-induced apoptosis, the JC-1 stain was applied to determine the mitochondrial membrane potential (Fig. 6). Following treatment with increasing concentrations of EEIHL, depolarized mitochondrial membrane potential gradually increased to $10.8 \%$ (Fig. 6A and B). Proteins determining mitochondrial fate were 
A

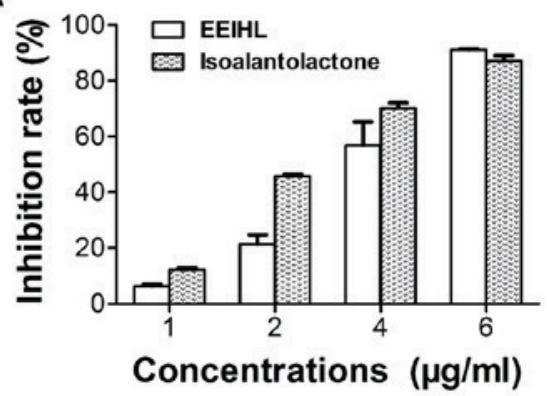

\begin{tabular}{c|c}
\hline CFPAC-1 & IC50 $(\mu \mathrm{g} / \mathrm{ml})$ \\
\hline EEIHL & $4.3 \pm 1.1$ \\
\hline Isoalantolactone & $2.8 \pm 1.3$
\end{tabular}

B

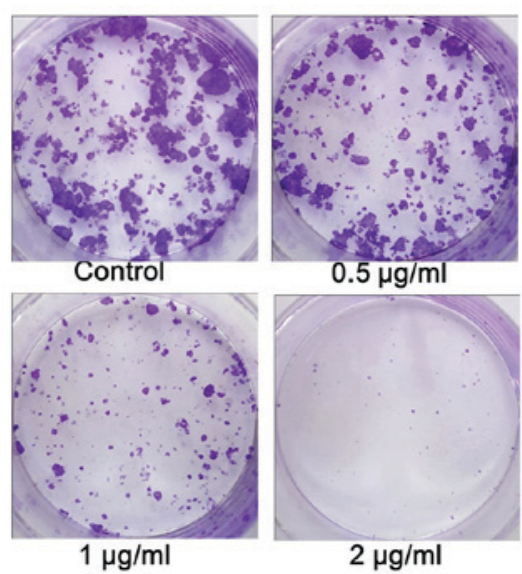

Figure 3. Anti-proliferative activity of EEIHL against CFPAC-1 pancreatic ductal adenocarcinoma cells. (A) A 48-h treatment of EEIHL dose-dependently inhibited the proliferation of CFPAC-1 cells. (B) CFPAC-1 cells were seeded into 6-well plates at a density of 1,000 cells/well and incubated for $24 \mathrm{~h}$, exposed to EEIHL for $48 \mathrm{~h}$, and cultured for a further 2 weeks. Cells were then fixed and stained with Giemsa solution prior to image capture. EEIHL, ethyl acetate extract of Inula helenium L.

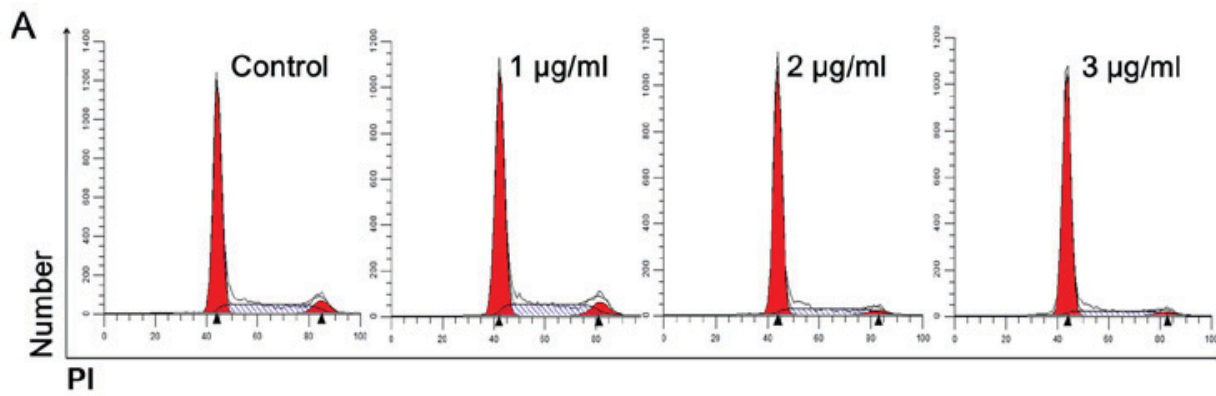

B

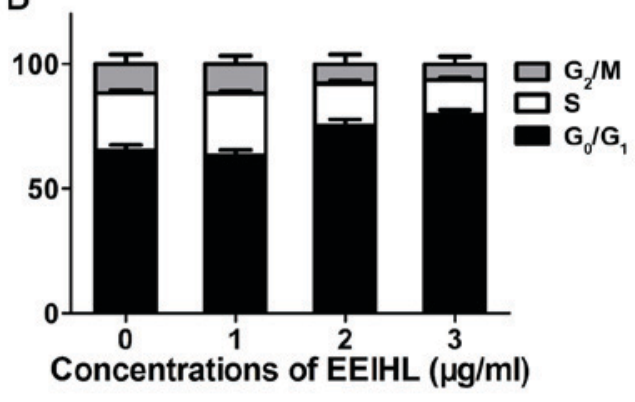

C

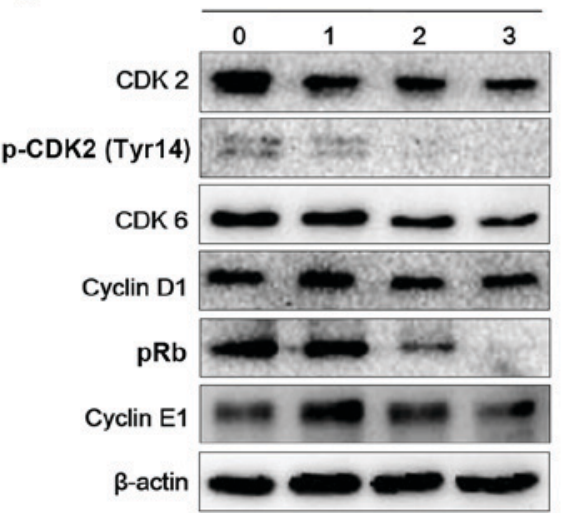

Figure 4. EEIHL induced $\mathrm{G}_{0} / \mathrm{G}_{1}$ cell cycle arrest in CFPAC-1 cells. (A) CFPAC-1 cells were seeded into 6 -well plates ( $2 \times 10^{5} /$ well) and cultured for 24 h. Cells were then treated with the indicated concentrations of EEIHL for $24 \mathrm{~h}$, followed by PI staining and flow cytometric analysis. (B) Quantitative analysis of cell cycle distribution. (C) Western blot analysis was used to analyze protein expression in CFPAC-1 cells following EEIHL exposure for $24 \mathrm{~h}$. EEIHL, ethyl acetate extract of Inula helenium L.; PI, propidium iodide; CDK, cyclin-dependent kinase; p-, phosphorylated.

then analyzed by western blot (Fig. 6D). In accord with the depolarized mitochondrial membrane potential, pro-apoptotic protein Bim was evidently elevated, whereas anti-apoptotic proteins Bcl-2 and Mcl-1 were downregulated, suggesting the crucial role of the mitochondria in EEIHL induced apoptosis. Furthermore, as indicated by the appearance of cleaved PARP and decreased levels of XIAP, EEIHL induced caspase-dependent apoptosis; this was verified by determining the caspase activity following EEIHL treatment (Fig. 6C). In addition, EEIHL induced the decreased expression of p-AKT and p-STAT3, suggesting alternative pathways involved in the anti-proliferation effect of EEIHL (Fig. 6D).

EEIHL inhibited CFPAC-1 cell migration. Cell migration was measured using a wound healing assay. When treated with 2,4 or $6 \mu \mathrm{g} / \mathrm{ml}$ of EEIHL, the migration ability of CFPAC-1 cells was gradually attenuated (Fig. 7A and B). In comparison with isoalantolactone, $4 \mu \mathrm{g} / \mathrm{ml}$ of EEIHL was significantly more potent in impeding CFPAC-1 cell migration. Snail is a transcriptional factor that promotes the 
A
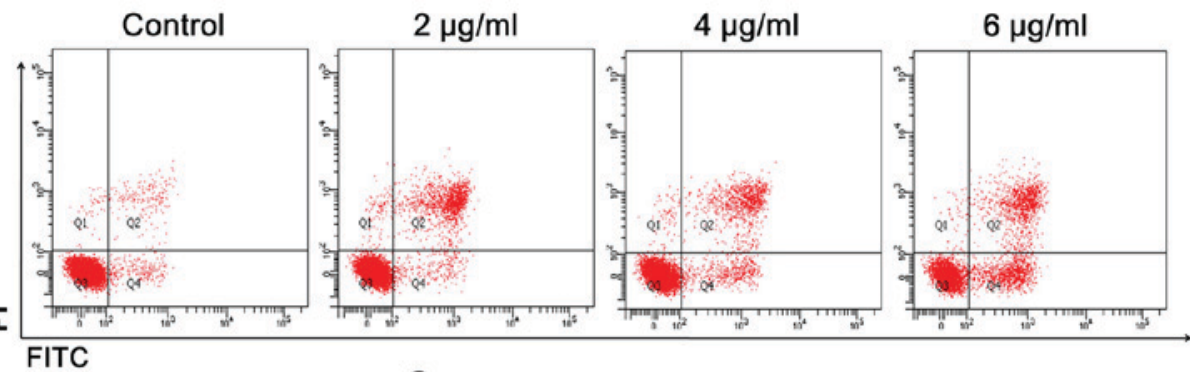

B

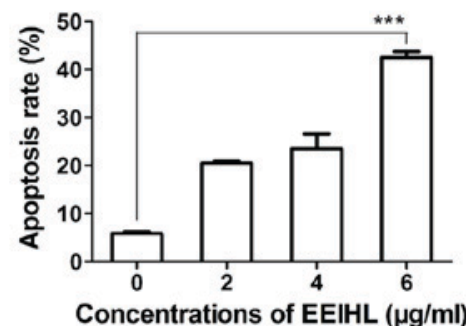

C

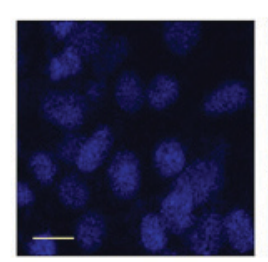

Control

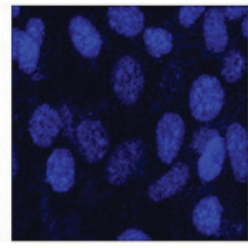

$2 \mu \mathrm{g} / \mathrm{ml}$

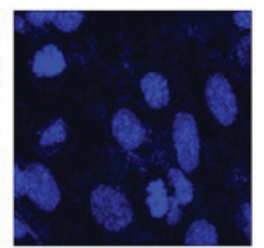

$4 \mu \mathrm{g} / \mathrm{ml}$

Figure 5. EEIHL induced apoptosis in CFPAC-1 cells. (A) CFPAC-1 cells were seeded into 6 -well plates (2x10 $/$ well) and cultured for 24 h. Cells were then treated with indicated concentrations of EEIHL for $48 \mathrm{~h}$, followed by Annexin-V/PI staining and flow cytometric analysis. (B) Quantitative analysis of apoptotic cells following EEIHL exposure. (C) CFPAC-1 cells ( $8 \times 10^{4}$ cells/well) were cultured in 24 -well plates. Following exposure to EEIHL, cells were fixed with $4 \%$ paraformaldehyde for $20 \mathrm{~min}$, and stained with DAPI for $15 \mathrm{~min}$. Scale bar, $20 \mu \mathrm{m}$. ${ }^{* * *} \mathrm{P}<0.001$. EEIHL, ethyl acetate extract of Inula helenium L.; PI, propidium iodide; FITC, fluorescein isothiocyanate.

A

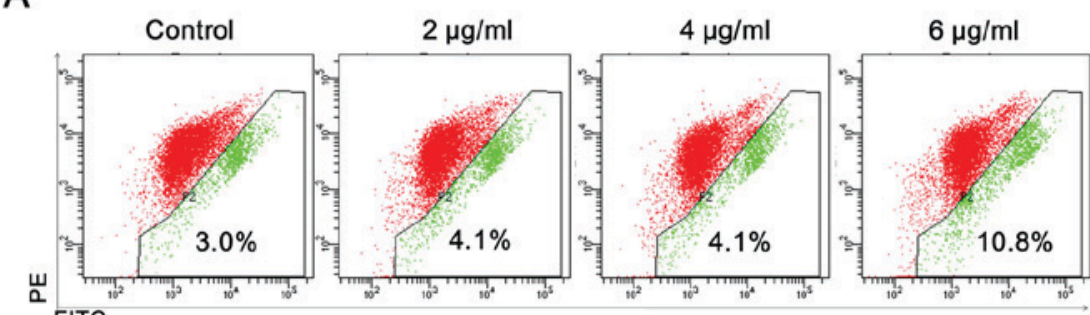

B
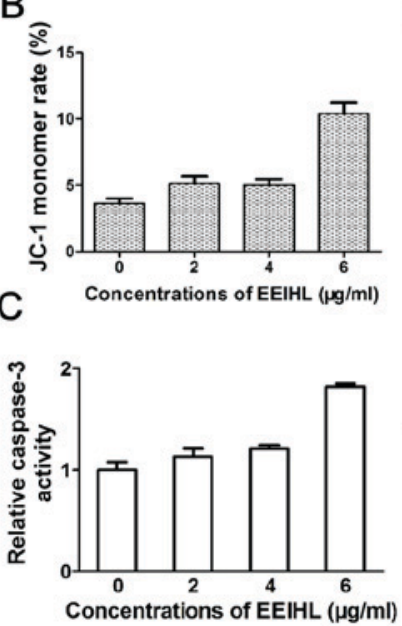

D

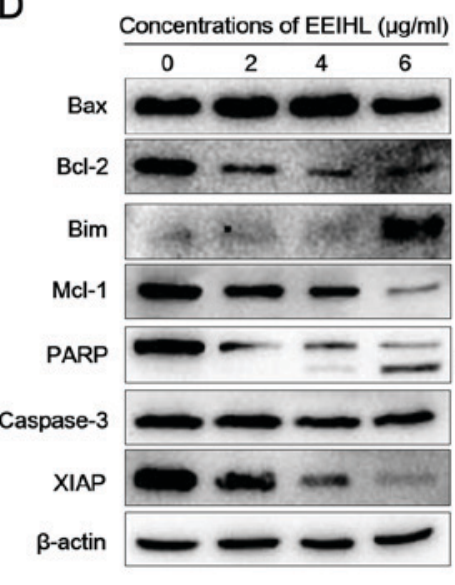

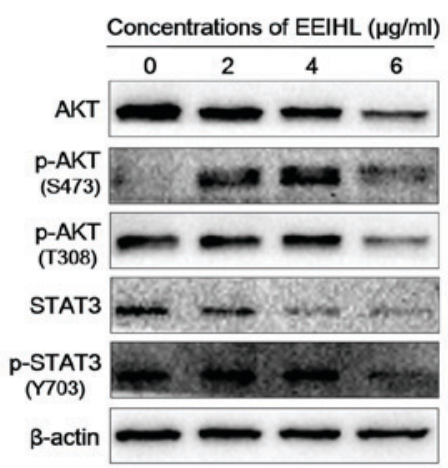

Figure 6. EEIHL promoted mitochondrion-dependent apoptosis. (A) CFPAC-1 cells were seeded into 6-well plates at a density of $2 \times 10^{5} /$ well, and cultured for $24 \mathrm{~h}$. Following treatment with EEIHL for $24 \mathrm{~h}$, the JC-1 stain was used to observe the depolarized mitochondria membrane potential by flow cytometry. (B) Quantitative analysis of depolarized mitochondria membrane potential. (C) CFPAC-1 cells were seeded into 6-well plates at the density of 2x10 $/$ well, and cultured for $24 \mathrm{~h}$. Following treatment with EEIHL for $48 \mathrm{~h}$, caspase-3 activity in cell lysates were determined with a colorimetric assay. (D) Following treatment with indicated concentrations of EEIHL for $48 \mathrm{~h}$, cells were lysed and proteins were extracted for western blot assay. EEIHL, ethyl acetate extract of Inula helenium L.; JC-1, 5,5',6,6'-tetrachloro-1,1',3,3' tetraethyl-imidacarbocyanine iodide; Bax, Bcl-2-like protein 4; Bim, Bcl-2-like protein 11; Mcl-1, Bcl-2-like protein 3; PARP, poly(ADP-ribose) polymerase; XIAP, X-linked inhibitor of apoptosis protein; STAT, signal transducer and activator of transcription; p-, phosphorylated.

repression of E-cadherin, which serves a key role in cell adhesion; the dysregulated transcription of Snail and E-cadherin is associated with the epithelial-mesenchymal transition (EMT) and tumor metastasis. As shown in Fig. 7C, the mRNA level 
A

A

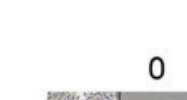

0
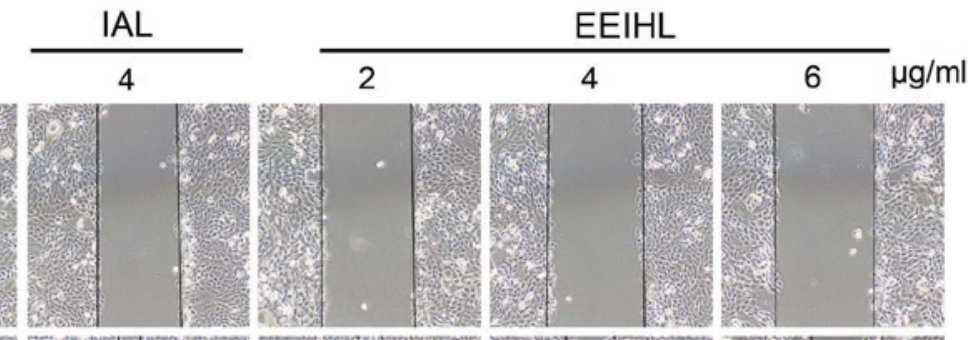

$\mathrm{Oh}$
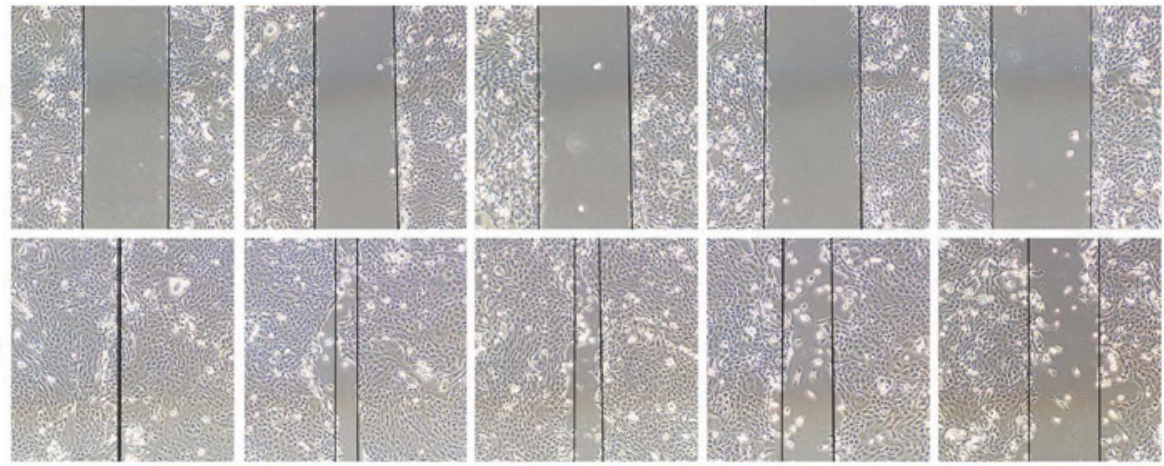

B

C
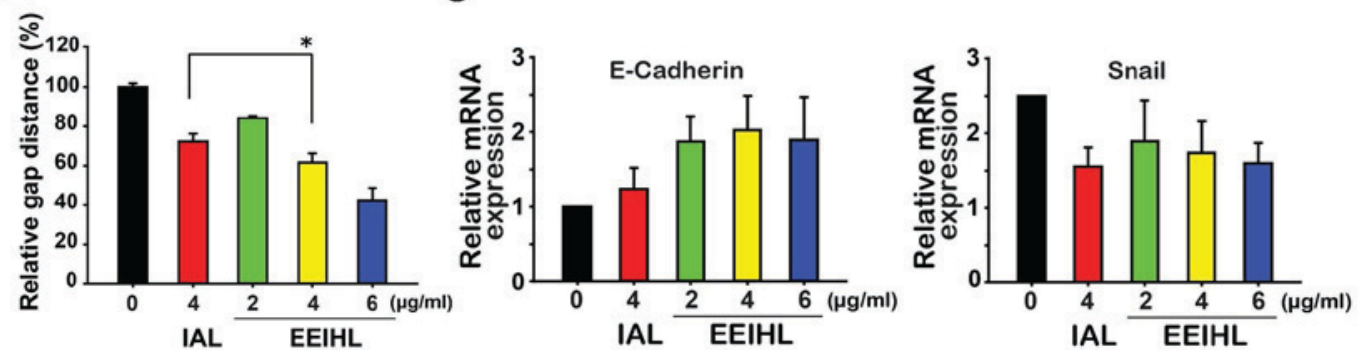

Figure 7. EEIHL inhibited CFPAC-1 cell migration through regulating epithelial-mesenchymal-transition-associated mRNA. (A) Cells were incubated for $24 \mathrm{~h}$ and were scratched by pipette tip across the center of the well. Following the removal of the detached cells, the remaining cells were allowed to grow for another $24 \mathrm{~h}$ before images were captured. (B) Quantitative analysis of wound lengths based on three independent experiments. (C) Following treatment with the indicated compounds for $24 \mathrm{~h}$, total RNA was extracted followed by reverse transcription-quantitative polymerase chain reaction. * $\mathrm{P}<0.05$. EEIHL, ethyl acetate extract of Inula helenium L.; IAL, isoalantolactone.

of E-cadherin increased following EEIHL treatment to a greater extent than treatment with isoalantolactone, indicating the inhibitory effect of EEIHL on cell mobility. Meanwhile, the mRNA level of Snail was significantly downregulated, suggesting that EEIHL may also inhibit the Snail-induced regulation of EMT.

\section{Discussion}

Inula helenium $\mathrm{L}$. is a traditional medicinal herb that may possess potent anti-cancer activity (5-7). As previously reported, sesquiterpene lactones are a class of chemical compounds responsible for the anti-cancer activity of IHL (17). Among those sesquiterpene lactones, alantolactone and isoalantolactone are the most extensively studied compounds obtained from the ethanol extracts of IHL $(18,19)$. However, there remain other sesquiterpene lactones that may also exhibit anti-cancer activity but have yet to be intensively studied (20). For instance, isocostunolide was identified as inducing caspase-dependent apoptosis in melanoma cells, and its $\mathrm{IC}_{50}$ value was comparable to alantolactone or isoalantolactone (21). The anti-PDAC activity and potential mechanisms of sesquiterpene lactones are not fully understood. The present study optimized the separation strategy by extracting IHL with $95 \%$ ethanol and partitioning with ethyl acetate, aiming to maximize the content of the sesquiterpene lactones that exhibit anti-cancer activity.

Sesquiterpene lactones extracted from IHL have been reported to inhibit proliferation through inducing apoptosis, causing $G_{1}$ phase arrest or activating reactive oxygen species (ROS) in several types of cancer, including breast cancer, leukemia and lung squamous cell carcinoma (22-24). To the best of our knowledge, there have been few studies regarding the effect on pancreatic cancer, apart from Khan et al (25) who identified that isoalantolactone could induce ROS-mediated apoptosis in PANC-1 cells. In the present study, EEIHL exhibited anti-proliferative activity against CFPAC-1 cells in a dose-dependent manner, without affecting the ROS level (data not shown) (26). Comparing the inhibitory effect of EEIHL with isoalantolactone, the $48 \mathrm{~h} \mathrm{IC}_{50}$ value of EEIHL on CFPAC-1 cells was $4.3 \mu \mathrm{g} / \mathrm{ml}$, a similar level to isoalantolactone $(2.8 \mu \mathrm{g} / \mathrm{ml})$. However, the inhibitory effect of impurities could not be excluded in this preliminary study. Future studies, should isolate the mixture of alantolactone, isoalantolactone and alloalantolactone, and further quantify the ratio of these three major components with antitumor activity, with the aim to determine the exact mechanisms of action of EEIHL-induced apoptosis. Additionally, one cell line is insufficient to represent the phenotype of metastatic pancreatic cancer. Accordingly, the authors of the present study are working on using additional pancreatic cell lines, including BxPC-3 and Capan-1, and HPDE6-C7 immortal human pancreatic duct epithelial cells, to demonstrate the specific antitumor effect of EEIHL on pancreatic cancer cells.

Low concentrations of EEIHL induced CFPAC-1 cell cycle arrest at the $G_{0} / G_{1}$ phase rather than in $S$ phase as in a previous study (27), indicating a distinct mechanism of action in CFPAC-1 cells. CFPAC-1 cells harbor allelic deletion in the SMAD4 gene, inducing the inactivation of 
SMAD4. Loss of SMAD4 causes alterations to multiple kinase pathways, including the p38 and AKT pathways, and increases chemoresistance in vitro $(28,29)$. The results of the present study indicated that high concentrations of EEIHL can downregulate the phosphorylation of AKT and STAT3, affecting mitochondrial apoptotic proteins, including Bim, Mcl-1 and Bcl-2. AKT is directly phosphorylated by PDK1 at T308, and forms an activation loop between SIN1, mTORC2 and p-AKT (T308), leading to the phosphorylation of AKT S473 by mTORC2 $(30,31)$. In addition, it has been reported that STAT/AKT pathway inhibition could cause apoptosis in prostate cancer (32). In the present study, it was identified that p-AKT (T308) and p-STAT3 (Y703) decreased following exposure to $6 \mu \mathrm{g} / \mathrm{ml}$ EEIHL, indicating STAT3/AKT inhibition following EEIHL treatment. To fully clarify the STAT3/AKT-associated mechanisms of EEIHL-induced apoptosis, it will be necessary to transfect cells with a hyper-phosphorylated STAT3 plasmid and knockdown AKT in a future study.

The present study compared the anti-migration activity of EEIHL with isoalantolactone by using a scratch wound healing assay. At a dose of $4 \mu \mathrm{g} / \mathrm{ml}$, a 24-h treatment with EEIHL demonstrated an anti-migration effect comparable to isoalantolactone. Additionally, EEIHL treatment significantly increased the mRNA level of E-cadherin, decreased the transcription of Snail, and downregulated the phosphorylation of STAT3 and AKT. Saitoh et al (33) identified that STAT3 acted as a mediator that could enhance the induction of Snail at the mRNA level. It was previously identified that the STAT3/AKT pathway mediated EMT and its downstream target, Snail, in hepatocellular carcinoma $(34,35)$. The function of STAT3/AKT/Snail in regulating EMT and thus, cell migration, has been established (36). Therefore, it was assumed that EEIHL could interfere with the phosphorylation of STAT3/AKT proteins, and downregulate the transcription of Snail, reducing the mobility of CFPAC-1 cells.

In conclusion, EEIHL produced anti-cancer activity in a concentration-dependent manner. Low concentrations of EEIHL induced $\mathrm{G}_{0} / \mathrm{G}_{1}$ phase arrest in CFPAC-1 cells, whereas high concentrations of EEIHL induced apoptosis, possibly via the downregulation of the phosphorylation of STAT3 and AKT. In addition, the inhibition of the STAT3/AKT pathway may affect downstream mRNA transcription, including of Snail and E-cadherin, leading to a reduced cell migration ability.

\section{Acknowledgements}

The present study was funded by Hangzhou Major Science and Technology Project (grant no. 20172016A01; to Nengming Lin), Zhejiang Provincial Program for the Cultivation of High-level Innovative Health talents (grant no., 2010-190-4; to Nengming Lin) and the National Natural Science Foundation of China (grant no., 81603144; to Bo Zhang).

\section{References}

1. Rahib L, Smith BD, Aizenberg R, Rosenzweig AB, Fleshman JM and Matrisian LM: Projecting cancer incidence and deaths to 2030: The unexpected burden of thyroid, liver, and pancreas cancers in the United States. Can Res 74: 2913-2921, 2014.
2. Bailey P, Chang DK, Nones K, Johns AL, Patch AM, Gingras MC, Miller DK, Christ AN, Bruxner TJ, Quinn MC, et al: Genomic analyses identify molecular subtypes of pancreatic cancer. Nature 531: 47-52, 2016

3. Garrido-Laguna I and Hidalgo M: Pancreatic cancer: From state-of-the-art treatments to promising novel therapies. Nat Rev Clin Oncol 12: 319-334, 2015.

4. Vincent A, Herman J, Schulick R, Hruban RH and Goggins M: Pancreatic cancer. Lancet 378: 607-620, 2011.

5. Seca AM, Grigore A, Pinto DC and Silva AM: The genus Inula and their metabolites: From ethnopharmacological to medicinal uses. J Ethnopharmacol 154: 286-310, 2014.

6. Stojanović-Radić Z, Comić LJ, Radulović N, Blagojević P, Denić M, Miltojević A, Rajković J and Mihajilov-Krstev T: Antistaphylococcal activity of Inula helenium L. root essential oil: Eudesmane sesquiterpene lactones induce cell membrane damage. Eur J Clin Microbiol Infect Dis 31: 1015-1025, 2012.

7. Qiu J, Luo M, Wang J, Dong J, Li H, Leng B, Zhang Q, Dai X, Zhang Y, Niu X and Deng X: Isoalantolactone protects against Staphylococcus aureus pneumonia. FEMS Microbiol Lett 324: 147-155, 2011.

8. Ghantous A, Gali-Muhtasib H, Vuorela H, Saliba NA and Darwiche N: What made sesquiterpene lactones reach cancer clinical trials? Drug Discov Today 15: 668-678, 2010.

9. Jiang HL, Chen J, Jin XJ, Yang JL, Li Y, Yao XJ and Wu QX: Sesquiterpenoids, alantolactone analogues, and seco-guaiene from the roots of Inula helenium. Tetrahedron 67: 9193-9198, 2011.

10. Zhang S, Won YK, Ong CN and Shen HM: Anti-cancer potential of sesquiterpene lactones: Bioactivity and molecular mechanisms. Curr Med Chem 5: 239-249, 2005.

11. Wang J, Zhao YM, Tian YT, Yan CL and Guo CY: Ultrasound-assisted extraction of total phenolic compounds from Inula helenium. ScientificWorld Journal 2013: 157527, 2013.

12. Park EJ, Kim YM, Park SW, Kim HJ, Lee JH, Lee DU and Chang KC: Induction of HO-1 through p38 MAPK/Nrf2 signaling pathway by ethanol extract of Inula helenium L. reduces inflammation in LPS-activated RAW 264.7 cells and CLP-induced septic mice. Food Chem Toxicol 55: 386-395, 2013.

13. Guo CY, Wang J, Hou Y, Zhao YM, Shen LX and Zhang DS: Orthogonal test design for optimizing the extraction of total flavonoids from Inula helenium. Pharmacogn Mag 9: 192-195, 2013.

14. Ahmed AF, Al-Qahtani JH, Al-Yousef HM, Al-Said MS, Ashour AE, Al-Sohaibani $M$ and Rafatullah S: Proanthocyanidin-rich date seed extract protects against chemically induced hepatorenal toxicity. J Med Food 18: 280-289, 2015.

15. Du J, Shi HR, Ren F, Wang JL, Wu QH, Li X and Zhang RT: Inhibition of the IGF signaling pathway reverses cisplatin resistance in ovarian cancer cells. BMC Cancer 17: 851, 2017.

16. Ding YL, Wang YZ, Zhang J, Zhang QZ, Zhang JY and Jin H: Application of the vanillin sulfuric acid colorimetry-ultraviolet spectrometry on quality evaluation of Panax notoginseng. Guang Pu Xue Yu Guang Pu Fen Xi 33: 471-475, 2013 (In Chines).

17. Konishi T, Shimada Y, Nagao T, Okabe H and Konoshima T: Antiproliferative sesquiterpene lactones from the roots of Inula helenium. Biol Pharm Bull 25: 1370-1372, 2002.

18. Weng Z, Gao H, Hu J, Fan Y, Wang H and Li L: Isoalantolactone induces autophagic cell death in $\mathrm{SKOV}_{3}$ human ovarian carcinoma cells via upregulation of PEA-15. Oncol Rep 35: 833-840, 2016.

19. Di W, Khan M, Rasul A, Sun M, Sui Y, Zhong L, Yang L, Zhu Q, Feng L and Ma T: Isoalantolactone inhibits constitutive $\mathrm{NF}-\kappa \mathrm{B}$ activation and induces reactive oxygen species-mediated apoptosis in osteosarcoma U2OS cells through mitochondrial dysfunction. Oncol Rep 32: 1585-1593, 2014.

20. Zaima K, Wakana D, Demizu Y, Kumeta Y, Kamakura H, Maruyama T, Kurihara M and Goda Y: Isoheleproline: A new amino acid-sesquiterpene adduct from Inula helenium. J Nat Med 68: 432-435, 2014.

21. Chen CN, Huang HH, Wu CL, Lin CP, Hsu JT, Hsieh HP, Chuang SE and Lai GM: Isocostunolide, a sesquiterpene lactone, induces mitochondrial membrane depolarization and caspase-dependent apoptosis in human melanoma cells. Cancer Lett 246: 237-252, 2007.

22. Zhao YM, Wang J, Liu HB, Guo CY and Zhang WM: Microwave-assisted Extraction of Alantolactone and Isoalantolactone from Inula helenium. Indian J Pharm Sci 77: 116-120, 2015. 
23. Cai H, Meng X,Li Y, Yang C and Liu Y: Growth inhibition effects of isoalantolactone on K562/A02 cells: Caspase-dependent apoptotic pathways, S phase arrest, and downregulation of Bcr/Abl. Phytother Res 28: 1679-1686, 2014.

24. Wang J, Cui L, Feng L, Zhang Z, Song J, Liu D and Jia X: Isoalantolactone inhibits the migration and invasion of human breast cancer MDA-MB-231 cells via suppression of the p38 MAPK/NF- $\kappa B$ signaling pathway. Oncol Rep 36: 1269-1276, 2016.

25. Khan M, Ding C, Rasul A, Yi F, Li T, Gao H, Gao R, Zhong L, Zhang K, Fang $X$ and Ma T: Isoalantolactone induces reactive oxygen species mediated apoptosis in pancreatic carcinoma PANC-1 cells. Int J Biol Sci 8: 533-547, 2012.

26. Zhang M,Hou Y, Shen Y, Guo X, Shang D and Zhang D: Probucol reverses homocysteine induced inflammatory monocytes differentiation and oxidative stress. Eur J Pharmacol 818: 67-73, 2018.

27. Yao Y, Xia D, Bian Y, Sun Y, Zhu F, Pan B, Niu M, Zhao K $\mathrm{Wu} \mathrm{Q}$, Qiao J, et al: Alantolactone induces G1 phase arrest and apoptosis of multiple myeloma cells and overcomes bortezomib resistance. Apoptosis 20: 1122-1133, 2015.

28. Chen YW, Hsiao PJ, Weng CC, Kuo KK, Kuo TL, Wu DC, Hung WC and Cheng KH: SMAD4 loss triggers the phenotypic changes of pancreatic ductal adenocarcinoma cells. BMC Cancer 14: 181, 2014

29. Zhang X, Cao J, Pei Y, Zhang J and Wang Q: Smad4 inhibits cell migration via suppression of JNK activity in human pancreatic carcinoma PANC-1 cells. Oncol Lett 11: 3465-3470, 2016.
30. Yang G, Murashige DS, Humphrey SJ and James DE: A positive feedback loop between Akt and mTORC2 via SIN1 phosphorylation. Cell Rep 12: 937-943, 2015.

31. Sarbassov DD, Guertin DA, Ali SM and Sabatini DM Phosphorylation and Regulation of Akt/PKB by the Rictor-mTOR Complex. Science 307: 1098-1101, 2005.

32. Guo Y, Zang Y, Lv L, Cai F, Qian T, Zhang G and Feng Q: IL-8 promotes proliferation and inhibition of apoptosis via STAT3/AKT/NF- $\kappa$ B pathway in prostate cancer. Mol Med Rep 16: 9035-9042, 2017.

33. Saitoh M, Endo K, Furuya S, Minami M, Fukasawa A, Imamura T and Miyazawa K: STAT3 integrates cooperative Ras and TGF- $\beta$ signals that induce Snail expression. Oncogene 35: 1049-1057, 2016.

34. Yu M, Xue H, Wang Y, Shen Q, Jiang Q, Zhang X, Li K, Jia M, Jia J, Xu J and Tian Y: miR-345 inhibits tumor metastasis and EMT by targeting IRF1-mediated mTOR/STAT3/AKT pathway in hepatocellular carcinoma. Int J Oncol 50: 975-983, 2017.

35. Kim HN, Narayanan NK, Lasano S and Narayanan B: Modulation of PGE2-induced EP4 expression on snail signaling and the impact on epithelial-mesenchymal transition: Significance of EP4 antagonism. Anticancer Res 31: 4347-4357, 2011.

36. Shi Y, Zuo D, Wang X, Han M and Wu Y: shRNA-mediated silencing of TARBP2 inhibits NCI-H1299 non-small cell lung cancer cell invasion and migration via the JNK/STAT3/AKT pathway. Mol Med Rep 14: 3725-3730, 2016. 